\title{
Geological appraisals of core samples using the ExoMars 2020 rover instrumentation
}

\author{
Keyron Hickman-Lewis ${ }^{\mathrm{ab}}$, Frédéric Foucher ${ }^{\mathrm{a}}$, Steven Pelletier ${ }^{\mathrm{c}}$, Fabio Messori ${ }^{\mathrm{d}}$, Frances \\ Westall $^{\mathrm{a}}$ \\ ${ }^{a}$ CNRS Centre de Biophysique Moléculaire, Rue Charles Sadron, 45071, Orléans, France \\ ${ }^{\mathrm{b}}$ Dipartimento di Scienze Biologiche, Geologiche e Ambientali (BiGeA), Università di \\ B ologna, Via Zamboni, 67, I 40126, Bologna, Italy \\ ${ }^{c}$ Université François Rabelais de Tours, Tours, France \\ ${ }^{\mathrm{d}}$ Università Degli Studi di Modena e Reggio Emilia, Modena, Italy
}

\begin{abstract}
The ExoMars 2020 mission will characterise a Martian locality with potential former habitability - Oxia Planum - and attempt to identify preserved physical and chemical biosignatures. The payload will include a drill retrieving cores from the subsurface (up to $2 \mathrm{~m}$ depth), which will be imaged at high resolution by two instruments: the Panoramic Camera High Resolution Camera (PanCam HRC) and CLose UP Imager (CLUPI). These instruments will provide guiding interpretation and govern the approach used by the analytical instruments, which will conduct their analyses after crushing of the core sample. Blind tests using Mars-analogue lithological samples provide valuable mission training in terms of maximising scientific return. Previous blind tests evaluating the abilities of ExoMars 2020 payload to conduct geological approaches used solid rock hand samples as test specimens. Here, we prepared samples of ExoMars mission-equivalent shapes and dimensions $(3 \times 1 \mathrm{~cm}$ cores). Imaging these samples using mission-equivalent original resolutions to avoid image processing artefacts, we found that the difficulty inherent in making definitive geological conclusions using traditional 'field petrography' approaches is increased when only limited amounts of core sample are available for observation. Issues inherent in interpreting core samples include scale-representativeness, distinction between layer-specific characteristics at the sub-centimetre to centimetre scale, mechanical effects, such as drill marks and dust covering, and correlation between analyses of the outcrop and the core. These issues vary depending upon the rock type and change as a function of the mechanical properties (and thus composition) of the sample. Despite these challenges, we find that CLUPI and PanCam HRC images alone allow many accurate and detailed geological observations; however, confidence and detail of interpretation are notably increased when additional geochemical data are provided, in this case, Raman spectra reflecting the contribution of the Raman Laser Spectrometer (RLS) instrument. Our results argue for the importance of core imaging during the experimental phase of the rover mission despite the challenges involved in interpretation, since HRC and CLUPI images offer a great degree of synergy. Inter-instrumental collaboration will be essential during the ExoMars 2020 rover mission (and indeed in any rover mission), since no single payload instrument is able to perform a comprehensive assessment of a putative biosignature within its geological context, and since the instrument suite provides highly complementary data at multiple scales that are key to maximising scientific return.
\end{abstract}




\section{1) Introduction}

\subsection{The geological context of Martian astrobiology}

The planetary-scale geodynamic decay responsible for the decline in Martian habitability since the Noachian period implies that missions aimed at the detection of traces of life should focus on geologically complex ancient terranes (Westall et al., 2015; Vago et al., 2017; Cabrol, 2018). If life emerged on Mars, it would likely have been restricted to the Noachian and early Hesperian, when conditions may have been broadly similar to those of the early Earth (Nisbet and Sleep, 2001; Westall et al., 2015). Much evidence supporting the long-term habitability of the surface Martian hydrosphere throughout the early history of the planet has been identified both from geomorphology and mineralogy. Geomorphological evidence is most apparent in the widespread dendritic river valley networks that extend across Noachian terranes (e.g., Malin and Edgett, 2003; Bhattacharya et al., 2005). Meandering channels indicate that Martian rivers were characterised by significant flows and long courses. Large bodies of standing water are indicated by the deltas impinging upon lakes and seas into which palaeo-rivers flowed (e.g. Pondrelli et al., 2005), although isolated lakes may have formed from meteoric water alone. Furthermore, a diversity in surface water states on the Noachian Mars is denoted by potential glacial deposits, including moraines and lobate debris aprons (e.g. Garvin et al., 2006), lineated valley floor deposits (Head et al., 2006) and ridges many kilometres in length interpreted as eskers (Butcher et al., 2017). Sedimentological and mineralogical evidence for aqueous episodes is abundant, the beststudied example being the kilometres of sediments observed by the MSL Curiosity rover at Gale Crater (e.g. Williams et al., 2013; Grotzinger et al., 2014; Eigenbrode et al., 2018). Deposits of hydrated minerals, including silica and hydrous phyllosilicates, are also common in many of the sedimentary deposits associated with the Martian hydrosphere (e.g., Wang et al., 2006; Chevrier and Mathé, 2007; Carter et al., 2013; Ehlmann and Edwards, 2014; Michalski et al., 2017). Enclaves for life associated with this hydrosphere would, under a trajectory of planetary-scale habitability degradation, have rapidly decreased early in Mars' history (Cockell, 2014), although recurrent periods of moisture may have occurred since the Hesperian (Kite, 2019). Identifying traces of ancient life fossilised billions of years previously poses a range of challenges. The unambiguous distinction of such traces of life in terrestrial rocks demands multi-technique investigations (e.g. Westall et al., 2011; Brasier et al., 2015; Wacey et al., 2016), which are more challenging on Mars given the inherent limitations of rover instrumentation. Thus, in order to maximise the chance of biosignatures detection at the landing site, several pre-requisites were necessitated:

i) the landing site must contain ancient strata with high habitability;

ii) studied samples should be taken from depth, avoiding surface degradative effects; and

iii) instrumentation should conduct an integrative, multi-scalar petrological, mineralogical and geochemical study at each studied locality.

\subsection{Martian astrobiology in the Age of Rovers}


The upcoming ExoMars 2020 (ESA-Roscosmos) and Mars2020 (NASA) rover missions ensure that the next decade will be formative in Martian astrobiology, both missions having objectives to search for extinct or extant traces of life (biosignatures) on Mars (Vago et al., 2006, 2017; Mustard et al., 2013; Hays et al., 2017). The instrumental payloads carried by both rovers are oriented toward the in situ analysis of biosignatures, and are capable of conducting petrological and organogeochemical assessments of biosignatures in their evolving geological environment (Vago et al., 2017; Cabrol, 2018). For technical reasons, the geological investigations on Mars do not follow the classical Earth protocol. There are no hammers permitting the observation of unaltered rocks, there are no possibilities for thin section preparation for mineralogy and micropaleontology, and many of the analyses will be carried out on crushed samples. These differences must be taken into account when interpreting the data collected on Mars (Foucher et al., 2013; Mangold et al., 2017). Emulations of mission operations - accounting for the constraints of space instrumentation and protocols - are therefore essential for training and learning processes in preparation for mission experimental cycles. Such training may use experimental setups mimicking the predicted environment (Martins et al., 2017) or the process of measurement (Foucher et al., 2013; Bost et al., 2015; this study).

Among the key targets of interest, microorganisms and organic molecules of unambiguous biological origin are of foremost importance (e.g., pigments, peptides). Organics have already been identified on Mars in Gale Crater sediments by the Mars Science Laboratory (MSL) Curiosity rover, firstly in the Sheepbed Mudstone at Yellowknife Bay (Freissinet et al., 2015) and later at the base of the Murray Formation at Pahrump Hills (Eigenbrode et al. 2018). Freissinet et al. (2015) identified hydrocarbons - 150-300 ppb by weight chlorobenzene and $70 \mathrm{ppb}$ by weight dichloroalkanes - that were determined to originate from the reaction of chlorine with organic carbon, whereas Eigenbrode et al. (2018) later identified thiophenic, aromatic, and aliphatic compounds with 50 nanomoles of organic carbon, the preservation of which may have been aided by sulphurisation. Nevertheless, although organic matter is evidently present at the surface of Mars, environmental, UV and ionising radiation has been shown to degrade most biological matter, as demonstrated by multiple experiments in laboratory and low Earth orbit, for example the experiments onboard the EXPOSE-E, EXPOSE-R and EXPOSE-R2 missions (Rabbow et al, 2012, 2015, 2017; de Vera et al. 2019 and references therein). Other authors have demonstrated that ionising irradiation may lead to the degradation of organics even at depth using laboratory setups (Kminek and Bada, 2006; Dartnell et al. 2012), and by numerical models (Pavlov et al., 2012). These studies concluded that, after several million years, a large proportion of the organic molecules of astrobiological interest would be degraded throughout the first tens of centimetres (down to more than 1.5 metres depth depending on the study). Thus, Kminek and Bada (2016) recommended drilling to at least $1 \mathrm{~m}$ to increase the potential of detecting biological remains dating to the Noachian-Hesperian period. On the other hand, geological processes may lead to the exhumation of rocks previously protected from irradiation by erosion, exposing well-preserved organic molecules at the surface. In light of this, Pavlov et al. (2012) advocated analysing rocks "freshly" exposed at the surface, in accordance with the sampling strategy used for the MSL mission. With the disappearance of liquid water activity at the surface of Mars, however, weathering and erosional processes have been very limited 
since the early Hesperian. Wind erosion and transport are also minor due to the low atmospheric pressure; therefore, very ancient geological structures can be observed at the surface (e.g. dried rivers dating back from the Noachian). Consequently, freshly exposed surfaces may be uncommon. Conversely, exposed rocks may have been "recently" covered by tens of centimetres of sand and dust, meaning that even if they are deeper than $1 \mathrm{~m}$ from the surface, the organics molecules they contain may have been destroyed before burial. To circumvent these issues, the ExoMars 2020 rover has the unique ability to drill down to $2 \mathrm{~m}$ in depth to collect samples, maximising the potential for the detection of well-preserved organic molecules (Vago et al., 2017). This method of collecting samples demands a unique investigative protocol that must be considered in the training for the mission.

\section{2) ExoMars scientific workflow and the importance of imaging systems}

\subsection{The ExoMars 2020 exploration protocol and scientific workflow}

The ExoMars 2020 rover is equipped with several external instruments: a highresolution imaging system, PanCam (Panoramic Camera) and an infrared spectrometer, ISEM (Infrared Spectrometer for ExoMars), located on the mast, a high resolution camera, CLUPI (Close-UP Imager), fixed on the drill box, and a neutron radiation detector, ADRON (Autonomous Detector of Radiation Of Neutrons), and radar for water detection and density and interface characterisation in the subsurface, WISDOM (Water Ice Subsurface Deposit Observation on Mars), both fixed on the body of the rover. The drill is equipped with a visible-near-infrared spectrometer, Ma_MISS (Mars_Multispectral Imager for Subsurface Studies), that will analyse the borehole wall. The drill will collect cores of $3 \times 1 \mathrm{~cm}$ that will be crushed ( $250 \mu \mathrm{m}$ grain size) prior to analysis by the instruments of the internal analytical laboratory: the Vis-IR spectro-imager MicrOmega, the Raman Laser Spectrometer (RLS), and a mass spectrometer, MOMA (Mars Organic Molecule Analyser). The positions of the instruments on the rover are indicated in Figure 1A. These instruments will enable the rover to carry out the exploration procedure described in Vago et al. (2017, pp. 496):

1- Landing site characterisation

2- Outcrop localisation, approach and study

3- Surface sampling (using the drill) and analyses

4- Subsurface characterisation to decide where to drill

5- Subsurface sampling and analyses 

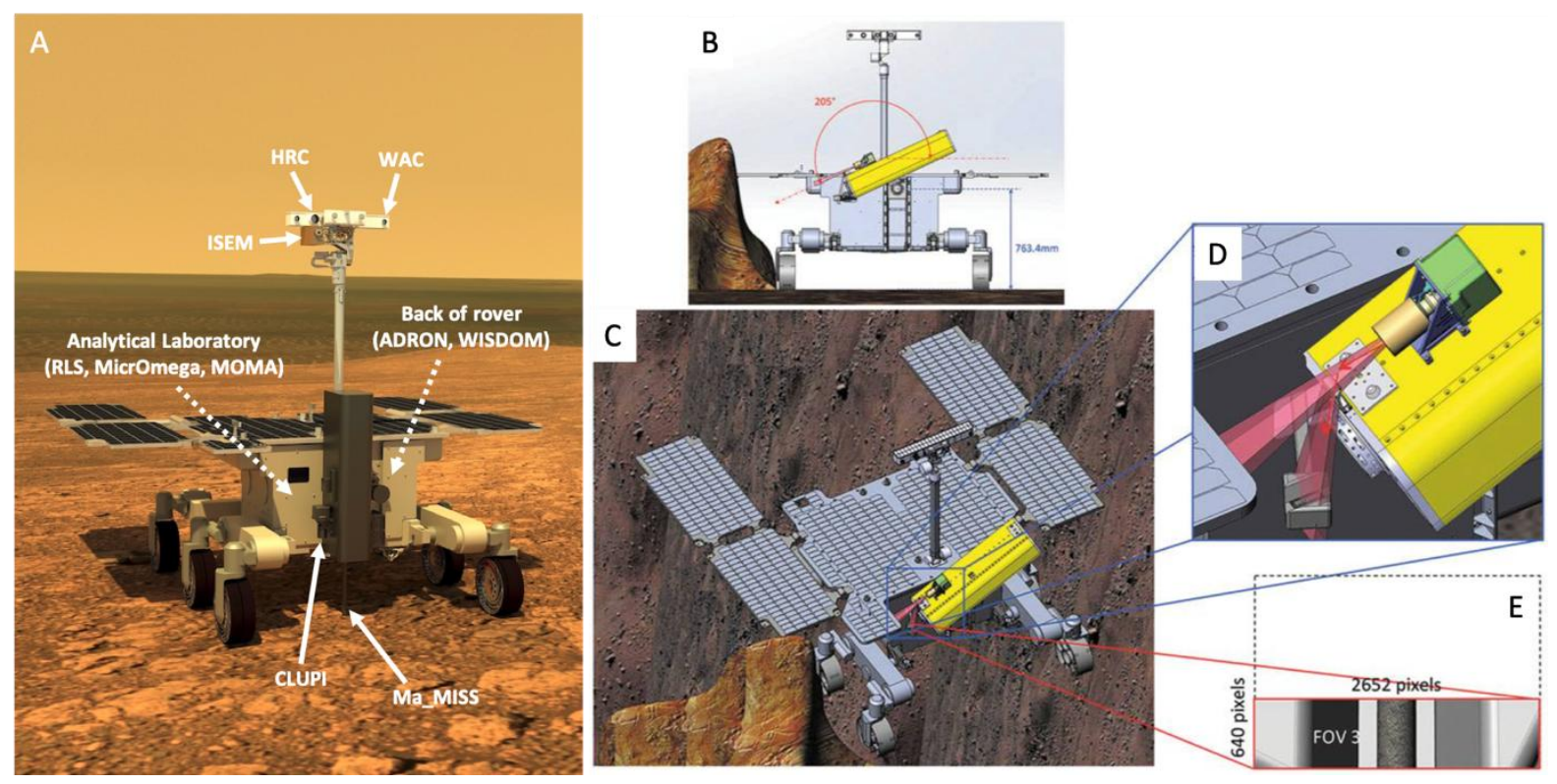

Fig. 1. ExoMars 2020 rover and aspects of its experimental cycle. A) Rendering of the ExoMars 2020 river showing the positions of the payload instruments (see main text for details). Credit: European Space Agency. B) Outcrop imaging configuration, drill arm shown in yellow. C) Top view of the outcrop imaging configuration with CLUPI shown in green mounted on the drill arm. D) Two CLUPI viewing modes, fields of view (FOVs) shown in red, CLUPI in green and the drill arm in yellow. FOV enables the imaging of core samples in the transport mechanism. E) Example image taken in CLUPI FOV3 mode. B-E adapted from Josset et al. (2017).

Accordingly, scientific data will be obtained following the specific workflow shown in Figure 2, involving progressively increasing resolution and a concomitant decrease in the scale of analysis. The findings of each step form a governing framework for the interpretation made in the next step (consistency). Similarly, at each step, there will be the opportunity to refine and/or reinterpreted the conclusions of the previous steps. This progressive learning approach is equivalent to that of MSL, described by Mangold et al. (2017). The interpretation of subsurface samples is, however, more complex due to the possible differences between surface and subsurface lithologies. Inconsistency between outcrop and subsurface samples must be considered and high-resolution data obtained from core analyses are limited in their utility for the refinement of surface data.

\subsection{The importance of imaging for Mars exploration}

Imaging is crucial for planetary exploration and all rovers and landers sent to Mars have been equipped with several imaging systems and cameras aimed at characterising the geological context and guiding the investigations (from punctual spectroscopic analyses to sampling and moving). High-resolution imaging systems are used to appraise the mineralogy, texture and structure of rocks and soils, similarly to field geologists using a magnifying hand lens. The MAHLI (Mars Hand Lens Imager) instrument on the Curiosity rover, for example, continues to play a major role in petrographic and mineralogical descriptions at Gale Crater (Grotzinger et al., 2014, 2015).

PanCam and CLUPI are the two external imaging systems onboard the ExoMars rover. PanCam, mounted on the rover mast (Fig. 1A), consists of two wide-angle cameras 
(WAC) for multi-spectral spectroscopic imaging and the High Resolution Camera (HRC) for detailed colour images. PanCam is the instrument primarily responsible for characterising the locality of the rover at outcrop scale, providing the 2D and 3D geological and mineralogical context for outcrops and the drilling site, in addition to navigation and strategic target identification along the rover traverses (Coates et al., 2017). Mast-mounted cameras have provided essential sedimentological and structural information in previous Mars missions, most recently the Mars Science Laboratory mission (Grotzinger et al., 2014, 2015; Rubin et al., 2017; Schieber et al., 2017). In addition to outcrop characterisation, HRC is also tasked with fine-scale outcrop characterisation, the monitoring of drilling operations and imaging the core during the short time that it is deposited in the core sample transport mechanism. CLUPI is a miniaturised, highly adaptive camera for high-resolution colour imaging (Josset et al., 2017), and is accommodated on the rover drill appendage (Fig. 1A-D). Two mirrors permit three fields of view (FOV): one for forward-facing observations of the geological environment and two for observation of the drilling area and drilled products (Fig. 1D-E). The first mirror provides an image of 2652 x 1768 pixels (FOV 1). The second mirror separates the field of vision and yields FOV 2, an image of 2652 x 1128 pixels in the direction of the optical axis of CLUPI, and FOV 3, $2652 \times 640$ pixels, oriented toward the drill tip. Thus, in addition to inspecting the local geological environment, CLUPI will provide the highest resolution images of drill core samples - before their subsequent crushing and ingestion into the body of the rover - using FOV 3 (Fig. 1E). In this configuration, CLUPI observes the core at a distance of $28.5 \mathrm{~cm}$ at an angle of incidence of $7.5^{\circ}$, ergo at a resolution of $22 \mu \mathrm{m} /$ pixel (Josset et al., 2017), capturing $1.42 \times 1 \mathrm{~cm}$ of the $3 \times 1 \mathrm{~cm}$ core sample. These images will provide a means of estimating rock type, mineral associations, context stratigraphy, grain size and shape parameters and distributions, material properties such as hardness, induration and friability, and secondary alteration phenomena (fractures, veins and voids). Sedimentary and igneous rocks are commonly characterised according to their grain size (Udden, 1914; Wentworth, 1922; Le Maitre, 2002). Figure 3 displays the type of rocks that could by identified by PanCam and CLUPI with respect to the working distance, considering that a resolution of at least $3 \times 3$ pixels/grain is required to determine the grain size. Sediment transport agents may also be determined, assisting in palaeoenvironmental reconstruction . A detailed study of grain morphology in CLUPI-representative images by Kapui et al. (2018) demonstrated the possibility to differentiate aeolian from fluvial sediments if the resolution is sufficiently high (approx. 30 x 30 pixel/grain).

Textural biosignatures down to the the millimetre- to micron-scale may also be imaged and, through appraising them concomitantly with secondary alteration phenomena, one may build a context scenario for signs of life. FOV 2 and FOV 3 will provide complementary information on rover activities, imaging the drilled area and the resulting deposits at the surface. A comprehensive description of the role of CLUPI in rover operations may be found in Josset et al. (2017) and Vago et al. (2017).

Fossilised terrestrial biosignatures are most easily identified using optical microscopy, however, on Mars, observations able to detect morphological biosignatures (e.g., biofilm stacks and microfossil colonies/aggregations) are conducted only during the imaging of outcrops and samples. Thus, for ExoMars, microscopic observations of core samples represent probably the only opportunity for in situ imaging of potential biosignatures. Since 
this contact phase of imaging (terminology of Vago et al., 2017) at scales between regional and mineral-molecule is designed to establish a large range of characteristic geological parameters for each sample, including habitability potential, the imaging phase of ExoMars operations - in which the notably synergistic approaches of the Close UP Imager (CLUPI) and Panoramic Camera High Resolution Camera (PanCam HRC) will be employed - would provide both guidance and context for fine-scale analytical studies (Vago et al., 2017; Josset et al., 2017; Coates et al., 2017). In this contribution, we simulate this synergy, applying a protocol through which we aim to:

i) determine the crucial observations made on core samples by coupling HRC and CLUPI imaging;

ii) assure confidence in these observations by coupling imaging data to mineralogical data (Raman spectra using a wavelength and data acquisition strategy comparable to that of the Raman Laser Spectrometer (RLS) instrument; Rull et al., 2017); and

iii) identify shortcomings in the observations made, thus evaluating the role of core imaging in the ExoMars 2020 imaging and experimental cycle protocols through a blind test. 


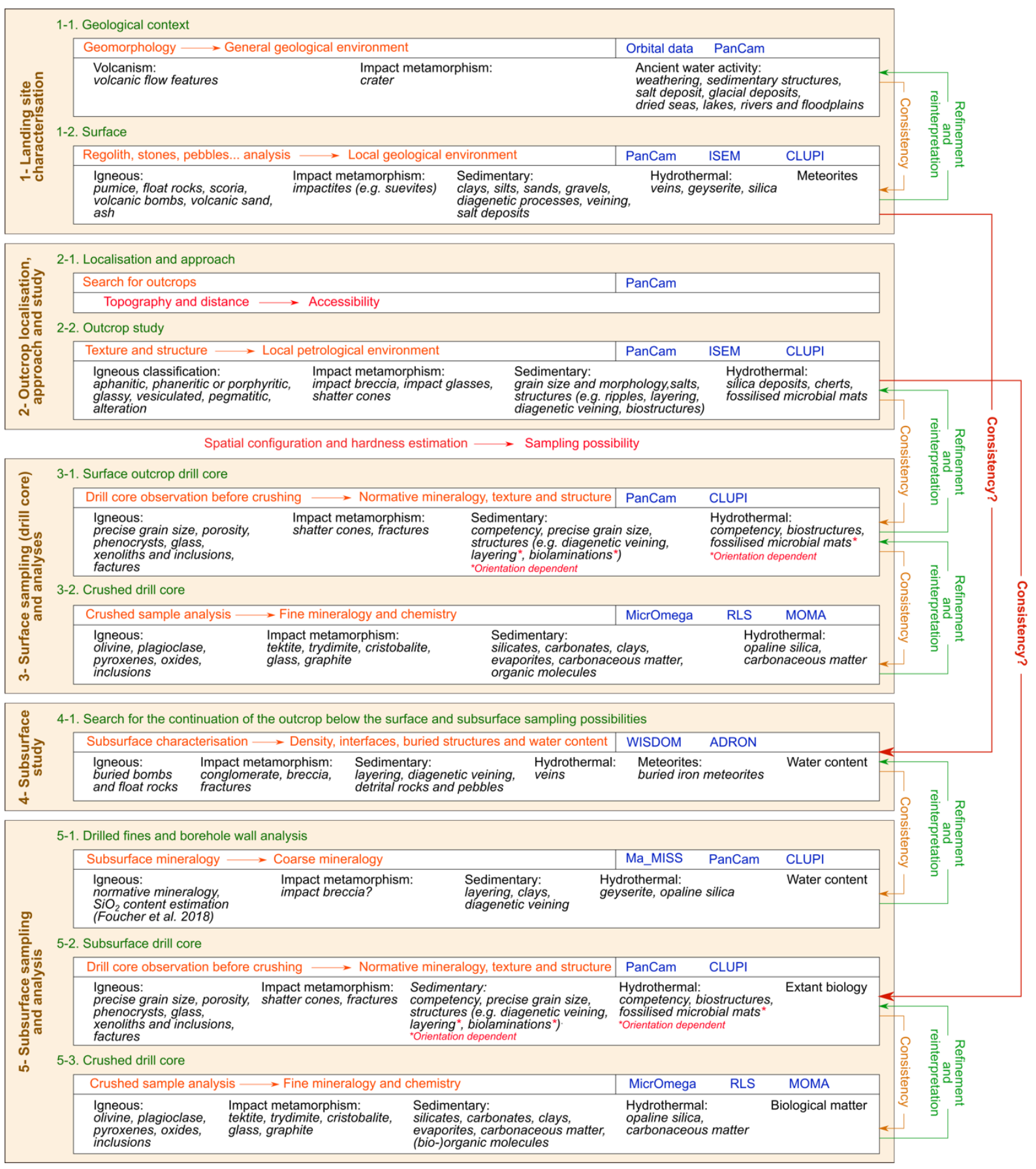

Fig. 2. ExoMars 2020 exploration procedure and scientific workflow. Comprehensive analysis of a locality involves three steps if only surface outcrops are studied, and five steps if subsurface samples are collected and analysed. Information in orange at each step describes the scope of analysis and the objective. Instruments performing these analyses are indicated at each step. Consistency of interpretation can be repeatedly checked after each stage of analysis, and reinterpretation made if necessary. Consistency between outcrop and subsurface analyses must be determined on a case-by-case basis, and requires detailed imaging of core samples. 


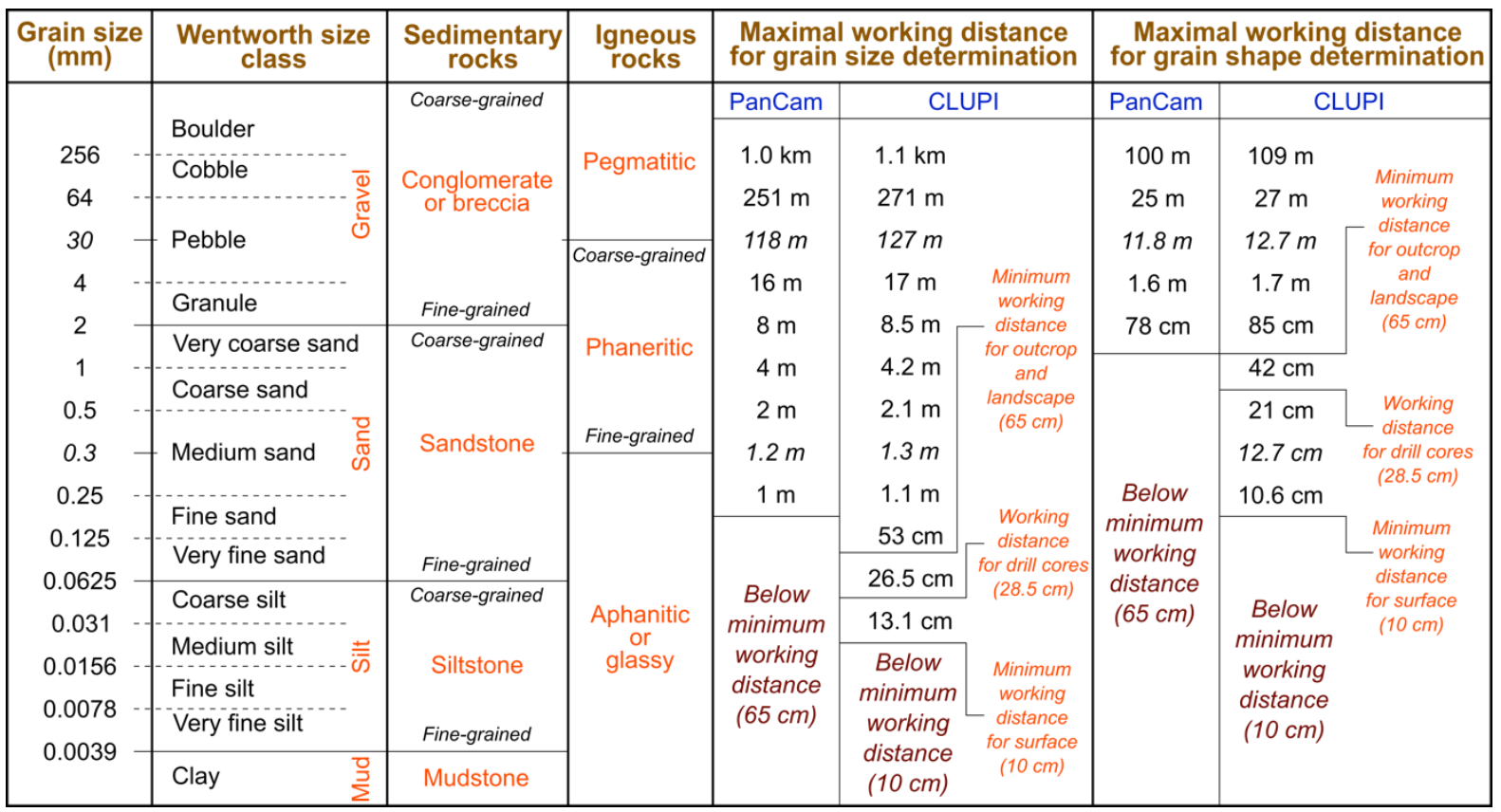

Fig. 3. Scales of sedimentary and igneous textures relevant to Martian geology, and the maximal working distance required to accurately identify them using PanCam or CLUPI. Working distances were obtained based on the fact that the ratio between the working distance and the resolution is equal to approximately 12,720 for CLUPI (Josset et al., 2017) and equal to 11,765 for PanCam (Coates et al., 2017), and considering that $3 \times 3$ pixels per grain are required to estimate grain size and that $30 \times 30$ pixels per grain are required to determine grain shape.

\subsection{Scientific and technical training using blind tests}

Bost et al. (2015) presented a first blind test using ExoMars 2020 instrumentation, studying two well-characterised samples from the International Space Analogue Rockstore (ISAR; Bost et al., 2013), a curated selection of astrobiologically relevant specimens. These were provided to imaging instrument teams as solid rock hand specimens with cut faces and to spectroscopy teams as powders, following which these teams were asked to attempt interpretation. Combining measurements acquired with CLUPI, Ma_MISS, MicrOmega and RLS emulator systems, it was found that comprehensive geological characterisation of outcrops could be achieved when combined with PanCam-representative context information from outcrops (of which the samples were representative). This was possible from optical images of hand samples alone, although much useful complementary information related to alteration features indicative of small scale habitability and potential biosignature preservation was added by the cross-correlation of data from the analytical geochemistry instruments. Nevertheless, the hand samples used by Bost et al. (2015) were around $10 \mathrm{~cm}$ in size and representative of the outcrop. Although this approach is relevant for drill cores taken at the surface of Mars, it is not necessarily the case for subsurface samples (see Fig 2., stage 5.2). For the test described herein, core samples of ExoMars 2020 dimensions $(3 \mathrm{x} 1 \mathrm{~cm})$ were prepared, and no outcrop information was provided. Consequently, all scientific interpretation presented in the blind test conducted herein is based entirely on the interpretation of core samples as imaged by HRC and CLUPI emulators, complemented by RLS-representative data. This approach more accurately evaluates the difficulties in 
interpretation resulting from limited sample volume and area of imaging, which may be sample- or lithology-specific. The objective of this blind test is therefore to assess - using mission-representative emulation of the core imaging process - to what extent geological observations can be made in the challenging scenario that core samples alone are available to characterise of the petrological context of putative biosignatures.

\section{3) Materials and Methods}

\subsection{Core samples}

Core samples were produced using specimens from the ISAR collection (Bost et al., 2013). Whereas the cores collected by ExoMars 2020 will be produced slowly and continuously, over a number of sols, without the use of water, our core samples were produced using water, the implications for which we outline in Section 4. Eight cores were produced, including volcanogenic, volcaniclastic, detrital sedimentary and biochemically precipitated rocks (Fig. 4; Table 1). Complete details of all samples are provided in Table 1. Cores measured $3 \mathrm{~cm}$ in length and $1 \mathrm{~cm}$ in diameter, i.e., they are dimensionally identical to those that will be produced during the mission.

Volcanogenic rocks (99SA13, 09SV17 and 17NZ14) were chosen to reflect some of the diversity in volcanic lithology identified on Mars (Bandfield et al., 2000; McSween et al., 2009). Detrital rocks (19AQ01, 11SP01, 17NZ05) were selected to reflect Martian sedimentary basin fills under erosive regimes. Oxia Planum, the chosen landing site for ExoMars 2020, is a phyllosilicate-rich terrain of volcanogenic and volcaniclastic materials sourced from volcanic terranes north of the ExoMars 2020 landing ellipse and their alteration products (Quantin et al., 2016). Due to the use of water in the coring process, the poorly indurated detrital rocks did not maintain cohesion; consequently, they were cut and shaped by manually grinding samples into the required dimensions in dry conditions. Two additional laminated sedimentary samples were chosen in order to test the ability of the imaging instrumentation to resolve millimetre-scale stratification, sedimentary structure and organicrich strata. Using these dimensionally relevant samples, we performed the test using the following protocol, elaborated upon in Sections 3.2-3.5, which simulates the core imaging phase and fundamental mineralogical characterisation by the analytical laboratory drawer.

i) Produce a FOV3 image of part of the core (identical resolution to CLUPI core imaging, e.g. Fig. 5) and conduct a preliminary lithological appraisal, noting any missing or indeterminable characteristics;

ii) Produce two HRC images at identical resolution to HRC core images (Fig. 6) in different lighting conditions.

iii) Acquire systematic Raman spectra to emulate RLS measurements. Re-appraise the sample and note any complementary information;

iv) Produce a high-resolution (CLUPI FOV3) image of the entire core sample (Fig. 4) and identify any further complementary information, i.e. the missing characteristics after mission-relevant steps i) and ii).

\subsection{CLUPI imaging}


CLUPI-representative images were acquired with a CLUPI emulator equipped with the same FOVEON detector as the flight instrument (SIGMA SD15 camera). CLUPI FOV3 is $5.90 \times 1.42 \mathrm{~cm}(2652 \times 640$ pixels, where each pixel is $22 \mu \mathrm{m})$, within which the core sample accounts for $450 \times 640$ pixels (Fig. 5). Focal length, aperture and working distance were set according to the objective used in order to obtain images of similar native resolution, i.e. meaning that no further image processing was applied to the photographs.

\subsection{PanCam HRC imaging}

The same SIGMA SD15 camera was used as an emulator for the HRC instrument. The HRC core images will be 1024 x 1024 pixels, where each pixel is $0.17 \mathrm{~mm}$, within which the core sample accounts for 177 x 59 pixels (Fig. 6). As for the CLUPI images, focal length, aperture and working distance were set according to the objective used in order to obtain images of similar native resolution, i.e. meaning that no further image processing was applied to the photographs.

\subsection{Raman spectroscopy}

The RLS instrument aboard ExoMars 2020 will use a $532 \mathrm{~nm}$ wavelength green laser to detect mineral phases in the crushed sample ingested into the rover analytical laboratory (Rull et al., 2017). RLS may use either an automatic (focussed on the RLS instrument) or cooperative (focussed on collaboration with MicrOmega and MOMA) operation mode. We simulated the automatic mode, acquiring twenty Raman spectra at consecutive, equally spaced intervals along the core samples. The system used was a WITec Alpha500 RA equipped with a frequency doubled Nd:YAG $532 \mathrm{~nm}$ green laser (similar to that of RLS) but using a spot size of $1.6 \mu \mathrm{m}$ diameter (i.e. smaller than the $50 \mu \mathrm{m}$ spot size diameter of RLS). Nevertheless, mineral identification using this system has been demonstrated to be representative of that conducted using the RLS system after data processing, spectral demixing and petrological considerations (Foucher et al., 2013).

\subsection{Method of questioning}

The protocol outlined in Section 2.1. was followed by a team of five geologists and one individual geologist, all of whom were involved in neither this project nor the ExoMars 2020 mission. The first step of the protocol determines whether core images taken by CLUPI (Fig. 5) alone are sufficient to identify the sample and, if not possible, at minimum to define certain characteristics (Table 2). The second step determines whether the various functions of PanCam HRC (Fig. 6) and RLS (identified minerals given in Table 1) can add critical information to validate and augment CLUPI information. This step also determines whether any identified characteristics are unreliable, not useful or whether further information is still needed (Table 3). The third step - high-resolution images of the full drill core (Fig. 4) confirms whether misidentifications are due to instrumental, material/dimensional or lithological factors (Table 3). The six geologists to whom we set this challenge - with expertise in approaches and techniques relevant to sedimentology, igneous petrology, geochemistry, geomorphology and palaeontology - were required to return the information pertinent to each step before being sent the data for the next step, such that interpretations were successively built on receiving more data, as during a mission. Subjects were asked not 
to make interpretations unless confident in so doing, determining which characteristics could be most accurately ascertained using specific instrumentation, and which would be unidentifiable without specific instrumentation.

\section{4) Results}

\subsection{SA13}

The CLUPI core image (Fig. 5A) allowed the geologists to describe 99SA13 (silicified volcaniclastics) as grey-blue, fine-grained and generally homogeneous, although no specific minerals were identifiable. The sample was correctly determined as well-indurated, with low porosity and high hardness. The presence of faint laminations independent of coring striations was noted, but the sedimentological character of these laminations could not be described in detail (simply described as depositional layers). Secondary fractures were noted. The geologists suggested that the rock was sedimentary (pelite). Small, reflective grains, scattered throughout the sample, were not identified, nor was the blotchy texture of the upper region of the image. HRC core images provided little complementary information, although they confirmed that the sample was well-indurated, not fragmented, and weakly stratified throughout (Fig. 6A). Raman spectra identified quartz, albite, orthoclase, epidote, muscovite and potentially titanite (Table 1), a mineral assemblage consistent with a metapelite of volcanogenic origin. This identification was accurate, and crucial observations were granulometry, stratification and mineralogy from both imaging and mineralogical data.

\section{2. $09 \mathrm{SV} 17$}

From the CLUPI core image (Fig. 5B), the sample appears green-yellow, mineralogically heterogeneous and granular. Suggested mineralogies included peridotitic minerals and their alteration products, and inclusions/xenoliths were identified (arrowed in Fig. 4B). The sample was deemed massive, non-porous, well-indurated, hard, and of magmatic origin. A preliminary identification suggested peridotite or serpentinite based on granulometry and estimated mineralogy. The addition of HRC core images did not add any complementary information, as the rock colour was too uniformly dark in tested lighting conditions to discern individual mineral phases (Fig. 6B). Raman data, however, proved extremely useful; a mineral assemblage of augite-diopside, enstatite, labradorite, forsterite, carbonaceous material and possible magnetite (Table 1) clearly suggest that this is a mafic (basaltic-gabbroic) rock. Full core images confirmed that the green mineralogy corresponded only to a coarse-grained xenolith, although the geologists did not identify the porphyritic fabric of the rock, leaving its petrogenetic history incompletely appraised; the rock is in fact a porphyritic basalt. The eventual interpretation was very accurate, in spite of some initial error, and colour, texture, xenoliths and mineralogy were deemed the key characters enabling the description of the sample (Table 1).

\section{3. $17 \mathrm{NZ14}$}

The CLUPI core image (Fig. 5C) enabled the immediate identification of a bipartite mineral assemblage: darker grey-black and lighter white-pink phases, which the geologists 
correctly suggested were mafic minerals and quartz/feldspars, respectively. The sample was also correctly characterised as coherent, hard, and rich in inclusions. The occurrence of stratification was mistakenly identified, since horizontal striations from the coring process sometimes coincided with zones of changing mineralogy. The geologists failed to identify the circular vesicles present throughout the sample (arrowed in Fig. 4C), as their depth was not obvious from the CLUPI image. Granulometry and the misidentification of stratification and vesicles resulted in the sample being incorrectly interpreted as a sedimentary conglomerate. HRC images did not assist the identification (Fig. 6C), since at their lower resolution, mineralogical zonation, which is broadly laminated in some parts of the rock, was misinterpreted as metamorphic foliation. Raman spectra again proved very useful for lithological interpretation: a pyroxene-rich mineralogy of mostly labradorite, some augitediopside, and minor anorthoclase feldspar and forsterite can only reflect a pyroxenite (Table 1). Thus, although the misindentification of foliation led to a final interpretation of metamorphosed pyroxenite, this revised interpretation is considerably more accurate than the preliminary suggestion of conglomerate. Mineralogical data enabled identification, however, the ambiguity of mineralogy in darker areas (vesicles) was a limiting factor to overall interpretation.
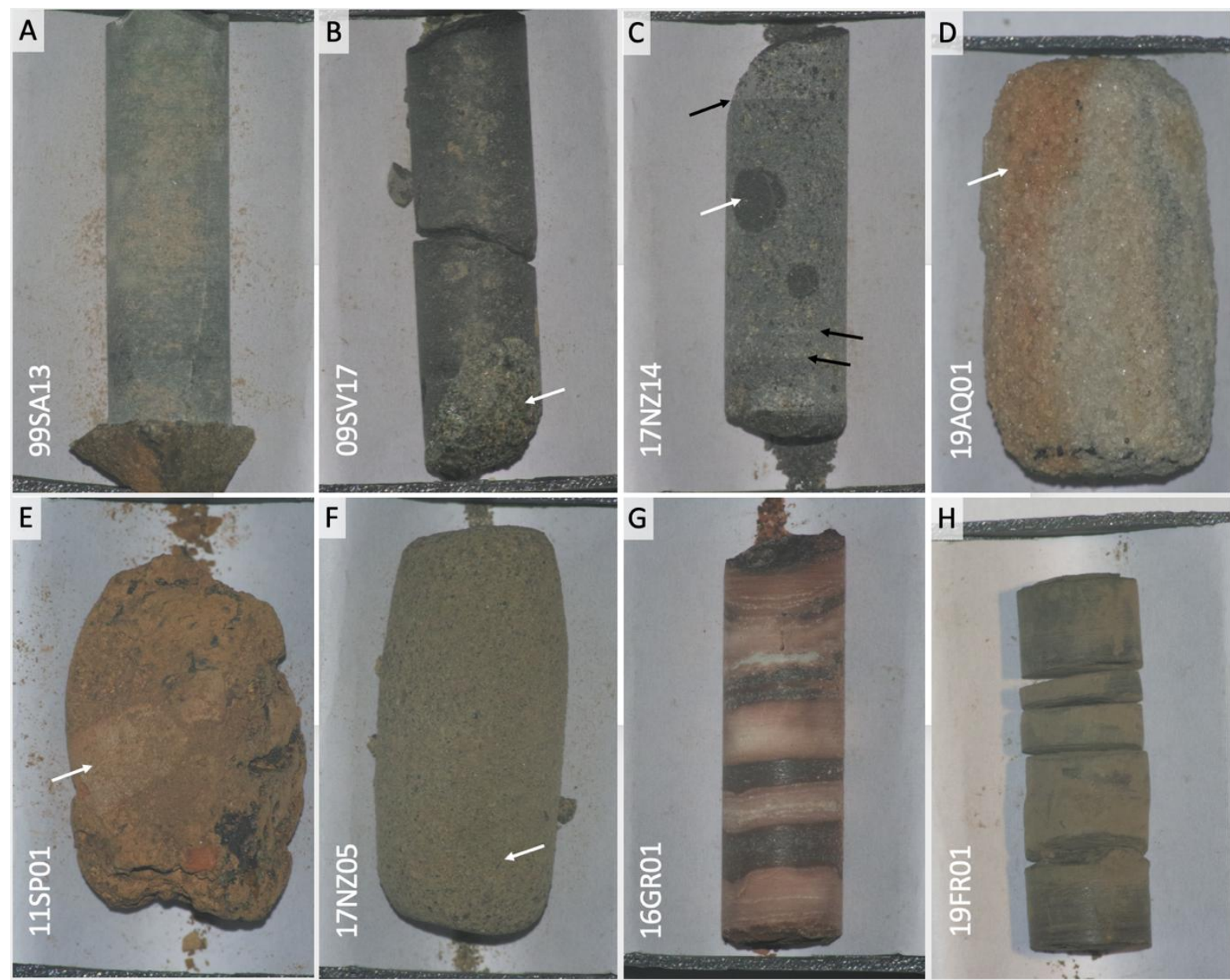

Fig. 4. Photographs (at maximal CLUPI resolution) of whole core samples, which are approximately $3 \mathrm{~cm}$ by $1 \mathrm{~cm}$ in size. A) Sample 99SA13, metapelite with carbonate. B) Sample 09SV17, tholeiitic basalt with copious large olivine-rich xenoliths (white arrow). C) Sample 17NZ14, basalt-pyroxenite featuring large spherical 
vesicles (white arrow). Damage during the coring process is reflected in surficial striations (black arrows). D) Sample 19AQ01, calcitic quartz arenite. White arrow indicates region of chemical alteration. E) Sample 11SP01, conglomerate with angular and sub-angular pale clasts (e.g., white arrow). F) Sample 17NZ05, coarse volcanogenic sandstone. White arrow indicates chevron-like black mineral. G) Sample 16GR01, ferruginous chert with alternately Fe- and Mn-rich layers. H) Sample 19FR01, finely laminated, organic-rich mudstone. The significance of the arrowed objects - causing difficulties in the interpretation - is given in the main text.
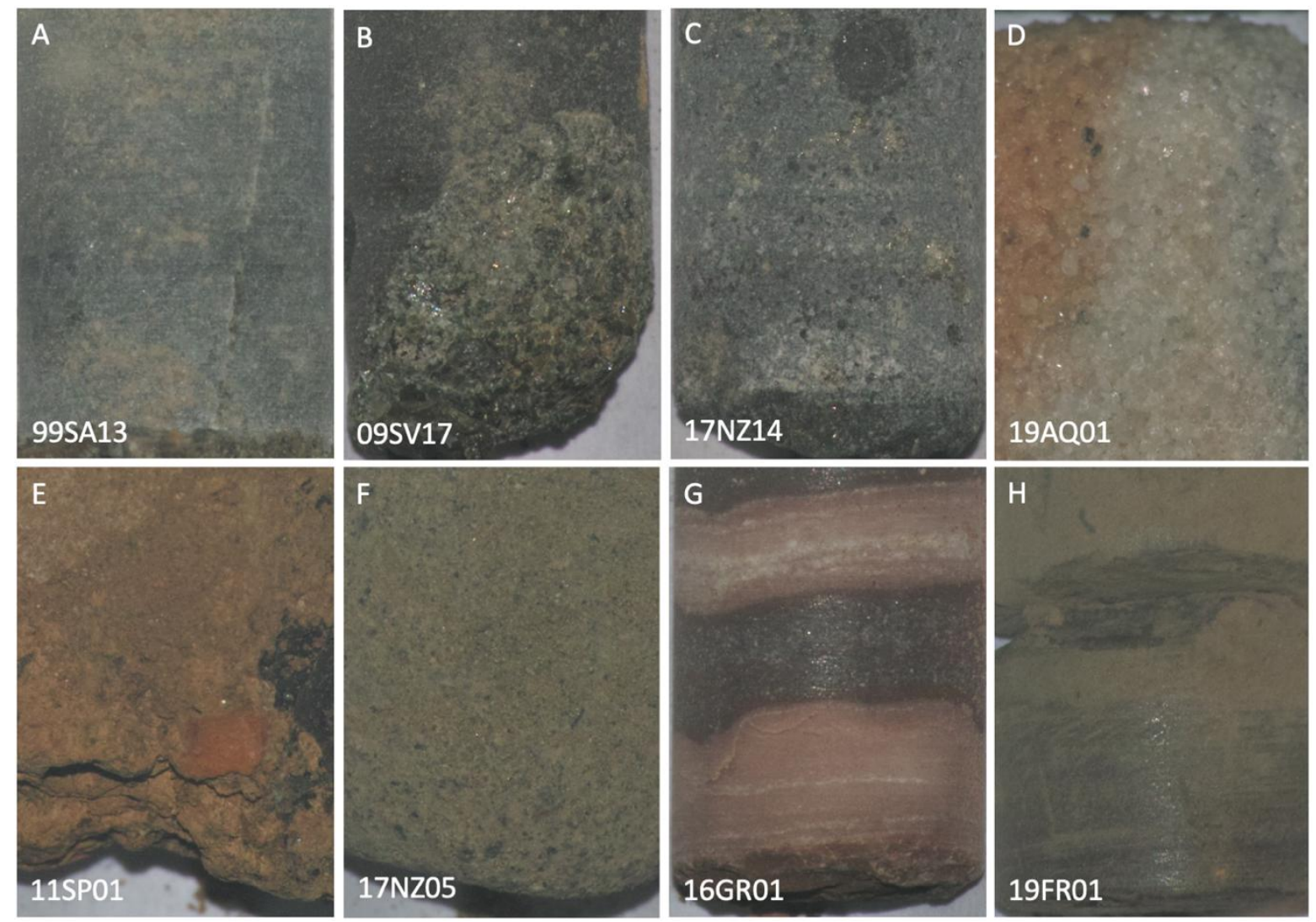

Fig. 5. Step 1 of the protocol: core samples of selected ISAR specimens imaged with emulated CLUPI FOV 3 (only core region shown) at maximal resolution of $22 \mu \mathrm{m} /$ pixel. Field of view is equivalent to that planned for CLUPI FOV 3 observations $(1.42$ x 1 cm). A) Sample 99SA13, metapelite with carbonate. B) Sample 09SV17, tholeiitic basalt. C) Sample 17NZ14, basalt-pyroxenite. D) Sample 19AQ01, calcitic quartz arenite. E) Sample 11SP01, conglomerate. F) Sample 17NZ05, coarse volcanogenic sandstone. G) Sample 16GR01, ferruginous chert. H) Sample 19FR01, finely laminated, organic-rich mudstone. See Table 1 for full lithological descriptions.

\subsection{AQ01}

The CLUPI core image (Fig. 5D) alone permitted a thorough examination of this coarse-grained quartz arenite. The geologists described the sample as orange, pink, white and bluish, coarsely granular, lightly porous, quite well-indurated and broadly homogeneous in terms of mineralogy (quartz) despite colour alterations. The rock was easily identified at this first step as a quartz-rich sandstone. HRC images and Raman spectra showing quartz, haematite, calcite and unidentifiable fluorescent minerals (Table 1) added no essential information but confirmed and added further detail to the identification (Fig. 6D). The sole remaining cryptic aspect was the vertical colour zoning (arrowed in Fig. 4D), although this was most easily explained as chemical weathering. The geologists did not comment on other 
characteristics of the grains (e.g. roundness, sphericity, kurtosis, skewness, Corey Shape Factors), despite the apparent ease of making these morphometric distinctions from the CLUPI core image. This sample was interpreted very accurately, with both granulometry from PanCam and CLUPI images, and mineralogy from Raman spectra providing all necessary information.

\section{5. $11 \mathrm{SP} 01$}

Although the CLUPI core image (Fig. 5E) of this iron-rich conglomerate allowed a number of sedimentary observations to be made, several crucial characteristics could not be confidently interpreted: hardness, stratification, porosity and mineralogy. The ochrous and black colouration of various zones of the sample were noted, as was the fine grain size and heterogeneity of the matrix. Comparison with other samples suggested that this was a more friable, poorly cemented lithology. Since no clasts were visible in the CLUPI image, an identification as a matrix-supported conglomerate was not possible. HRC images (Fig. 6E) showed the conglomeratic characteristics of the sample (large entrained particles in a fine matrix), however, large, angular, pale grains (arrowed in Fig. 4E) were misinterpreted as secondary alteration features. Raman data, identifying haematite (the surface alteration), quartz, the zeolite chabazite, carbonaceous material and possible magnesiochromite (Table 1), led to an eventual inaccurate and incomplete identification as a detrital iron-rich sediment, tentatively a ferruginous laterite.

\section{6. $17 \mathrm{NZ} 05$}

From the CLUPI core image (Fig. 5F), the geologists described a green-grey, heterogeneous, granular rock, both well-indurated and cohesive, with no distinct sedimentary features. Elongate, prismatic black minerals were interpreted as amphiboles. Lighter, reflective minerals (oxides) were not noted, but chevron-like black minerals were identified throughout the sample. At this stage, the geologists were not confident in making an identification. HRC images (Fig. 6F) showed the mineralogical diversity at different scales of observation: whereas at the scale of tens of microns (step one), the sample is heterogeneous, at the scale of millimetres, the sample is homogeneous (step two) and structureless. In HRC images, the sample appears brown, as opposed to green-grey (Fig. 6F). Only two Raman spectra were obtained, corresponding to leucite/labradorite and anorthoclase/labradorite, i.e., the rock contains calcium feldspar (Table 1). All other spectra were masked by high fluorescence, but a magmatic origin was suspected based on these two measurements. Without identification of the clay mineral cementing phase (achievable using Ma_MISS and MicrOmega), and in the absence of visible sedimentary structures, the logical steps leading to an identification as volcanogenic sandstone could not be made. The eventual interpretation was therefore broadly accurate, but lacked many important details. The geologists noted that colour and certain unidentifiable phases (the matrix and numerous chevron-like minerals; arrowed in Fig. 4F) made the appraisal particularly challenging.

\subsection{GR01}

The CLUPI and HRC core images (Figs. 5G and 6G) allowed an equally comprehensive petrographic and petrological description of this banded ferruginous chert. 
Heterogeneous laminations alternate between grey-black and pink-orange-white colouration and exhibit differential coarseness, dark laminations being coarser. The rock is evidently hard and well-indurated, with pressure solution fronts (stylolites) at lamination boundaries. In this first step, the geologists speculated that evaporite minerals might constitute the darker layers, and that the rock itself may be a biochemical precipitate. HRC permitted observations on the millimetre scale and aiding the visualisation of vertical variation in lamination. Raman data identified almost exclusively haematite, with minor orthoclase and microcline (Table 1). Although the composition of the dark bands remained difficult to interpret, having apparently not been measured during the Raman analyses, comparison with similar strata from the geological record allowed accurate determination that this is a banded ferruginous chert, and therefore reflects (bio-)chemical deposition.
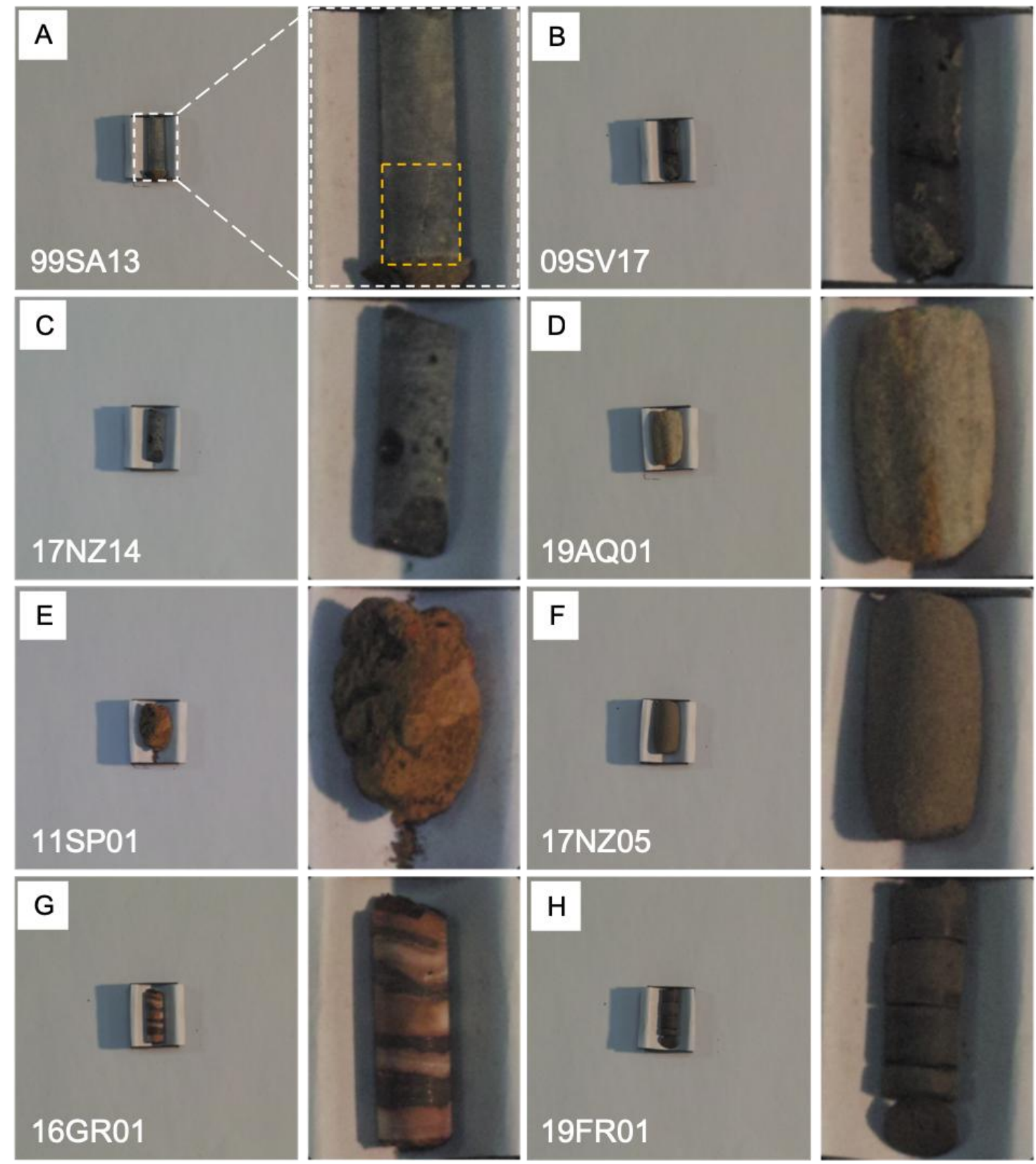
Fig. 6. Step 2: selected core samples imaged with HRC emulator conditions at a resolution of $17 \mathrm{~mm} / \mathrm{pixel}$. Samples were each imaged in two light conditions. Field of view is equivalent to that planned for HRC images $(17.4 \times 17.4 \mathrm{~cm})$. Yellow box in A indicates size of the FOV3 of CLUPI (cf. Fig. 3A). A) Sample 99SA13, metapelite with carbonate. B) Sample 09SV17, tholeiitic basalt. C) Sample 17NZ14, basalt-pyroxenite. D) Sample 19AQ01, calcitic quartz arenite. E) Sample 11SP01, conglomerate. F) Sample 17NZ05, coarse volcanogenic sandstone. G) Sample 16GR01, ferruginous chert. H) Sample 19FR01, finely laminated, organicrich mudstone.

\subsection{FR01}

The CLUPI and HRC core images (Figs. 5H, 6H) both show a brown-grey-yellow, fine-grained, heterogeneous sample, which is cohesive but soft relative to the other studied samples. Stratification was identified independent of striations left by the coring mechanism, however, porosity was mistakenly inferred where none exists. Dust from the fragmentation of the sample further hindered observation of the upper part of the image; nonetheless, this sample was correctly identified as a fine-grained, organic-rich, laminated sediment (mud- or silt-grade) from CLUPI and HRC images alone. The sole difference between the two images is that the colour variation seen in the CLUPI image is an artefact of the small area of observation; the wider scale of observation of the HRC image demonstrates that the sample is colourimetrically and compositionally repetitive. Raman measurements identify mostly carbonaceous material, often associated with quartz and brookite $\left(\mathrm{TiO}_{2}\right)$, together with minor haematite and zircon, i.e. a siliciclastic composition (Table 1). The geologists made an accurate identification of the sample and deemed that the fine grain size, regular, fine-scale stratification and compositional information were equally important characteristics for successful interpretation.

\section{5) Discussion}

5.1. Differences inherent in the study of samples on Earth and Mars

Although all reasonable effort was made to ensure the mission-representativeness of our experiments, a small number of technical process differences between the study of samples in Earth and Mars conditions are unavoidable:

- During the ExoMars 2020 mission, no water will be used for drilling. This will increase dust production, which may hinder the visual interpretation of samples.

- On Mars, drilling will be conducted very slowly and smoothly leading to fewer and less distinct striations, which reduces the risk of misinterpretation of such features.

- Martian light conditions have not been considered in the test. The difference in light colour and intensity may change the apparent colour of samples and influence interpretation. In light of this, image calibration methods permitting the correction of sample colour on Mars are presently under development (Josset et al., 2017; Foucher et al., 2018).

- No liquid water is expected in the exhumed samples. In rocks which exhibit cementation as a function of water content, e.g. sandstones, this may alter competency and increase friability. 
- The exhumation of core samples at the Martian surface could induce the sublimation of water ice, causing the cracking and disaggregation of samples with closed porosity, thus the core samples may emerge with their stratigraphy disturbed.

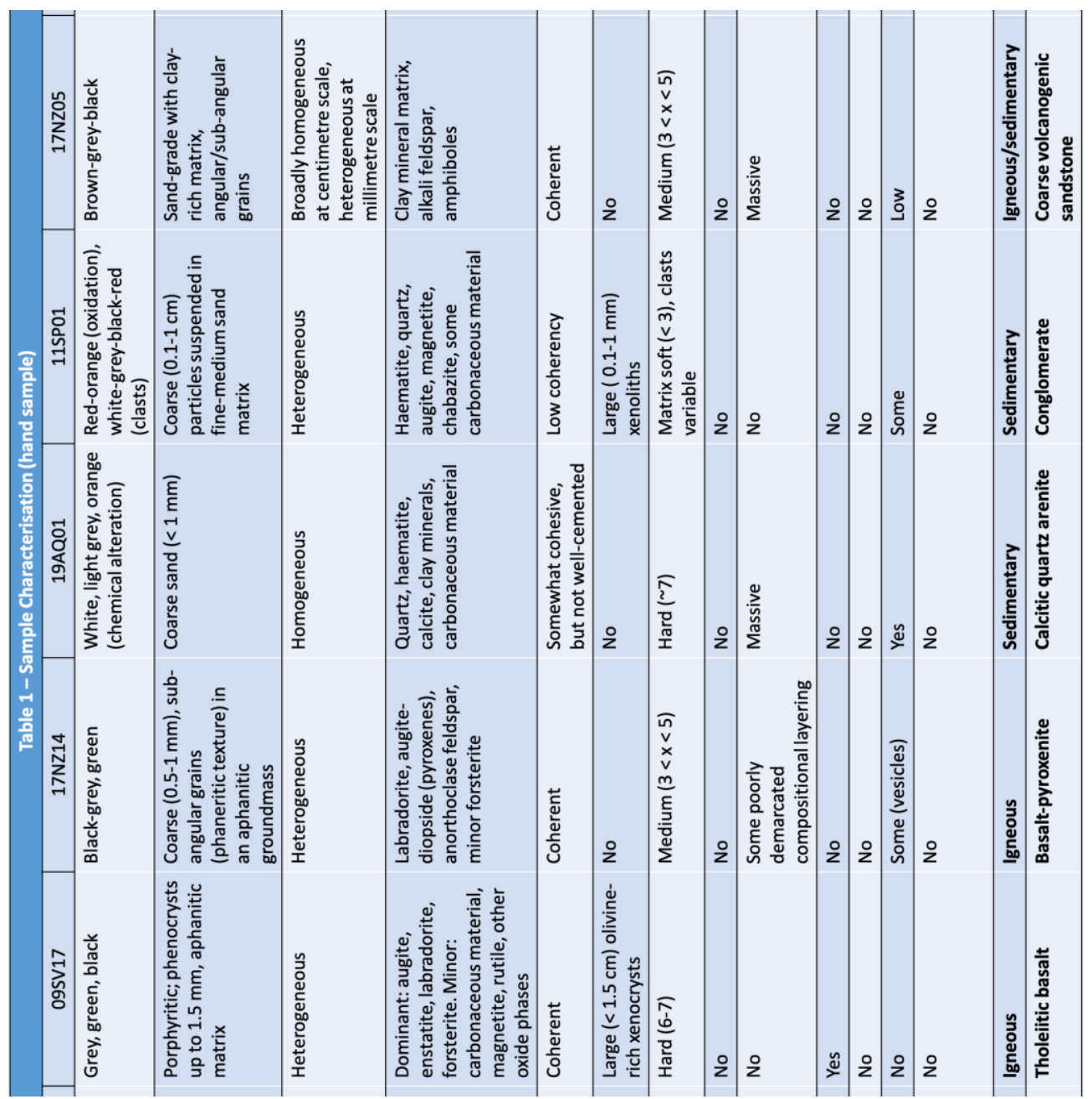

Table 1. Geological and petrological descriptions of the eight samples used in the blind test. 


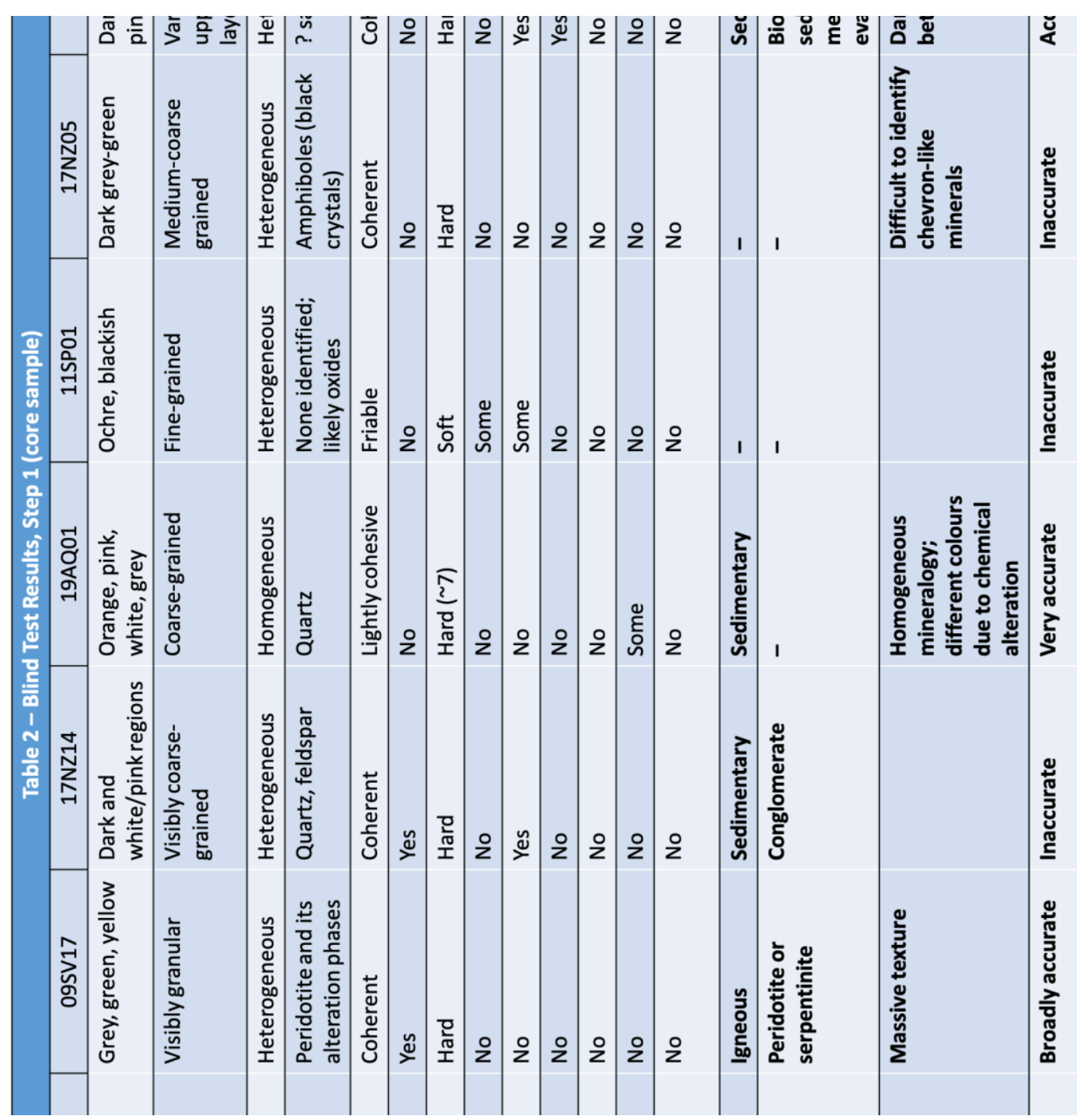

Table 2. Responses of the subjects after step 1 of the protocol described in Section 2.1. 


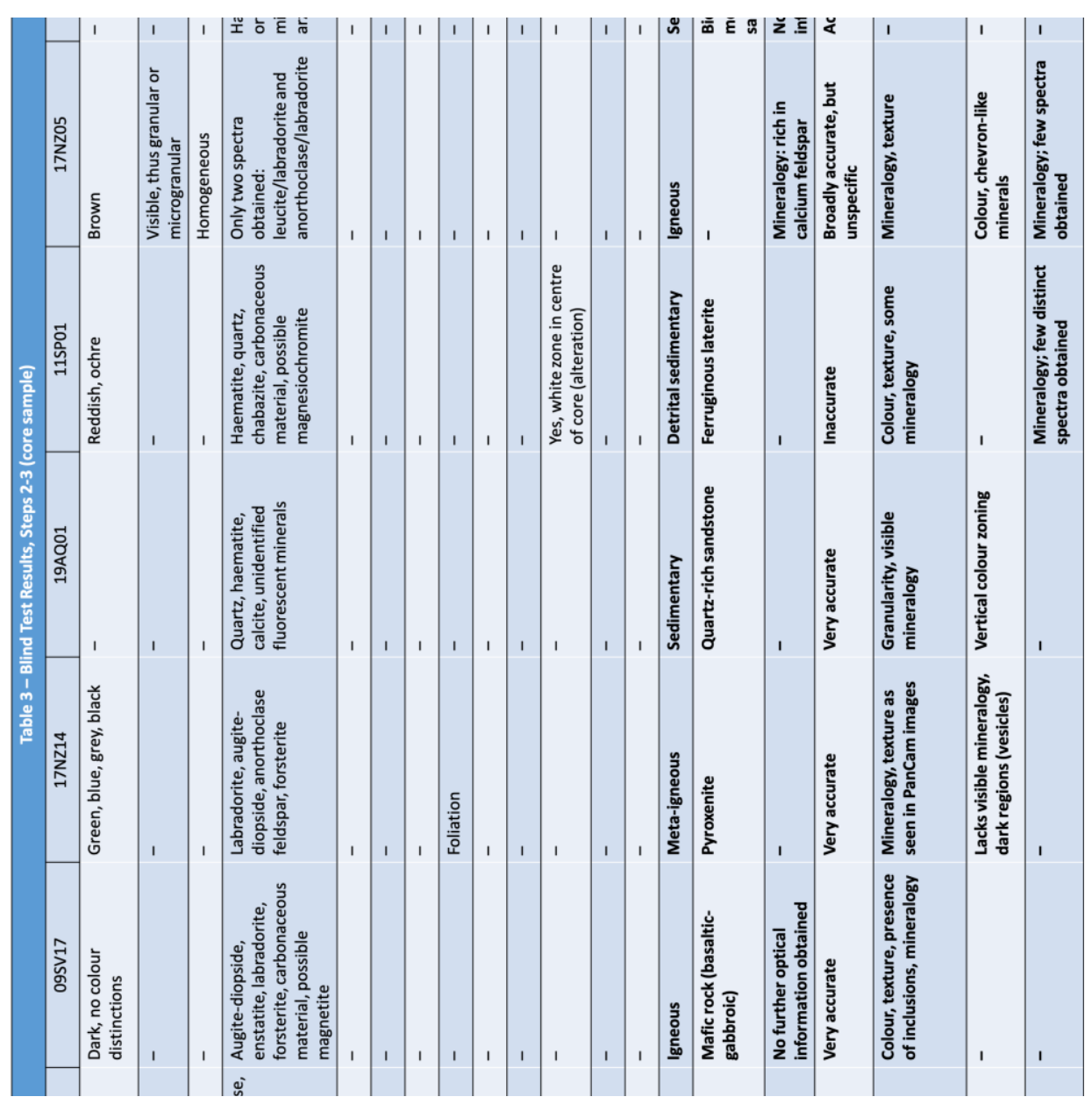

Table 3. Responses of the subjects after steps 2-3 of the protocol outlined in the Section 2.1. Where '-' is indicated, no further conclusions could be made with respect to those already made after step 1, i.e. CLUPI alone offered as much information as was possible or necessary.

\subsection{Challenges of interpreting limited samples}

\subsubsection{Misidentification and misinterpretation}

The principal challenge inherent in the interpretation of core samples is the possibility of misinterpretation of features at the sub-centimetre to centimetre scale. Although these features are within the range observable by the ExoMars 2020 imaging instrumentation (see Fig. 3), their interpretation is more difficult in practice, particularly given the limited field of view possible for core samples. CLUPI images only $1.42 \mathrm{~cm}$ of the length of the core at 22 $\mu \mathrm{m} /$ pixel (Josset et al., 2017). Using HRC, the whole core is imaged with a resolution 0.17 $\mathrm{mm} /$ pixel (Coates et al., 2017), thus the sample accounts for only 177 x 59 pixels (the full image being 1024 x 1024 pixels, corresponding to 17.4 x $17.4 \mathrm{~cm}$ ). Consequently, sedimentary structures (e.g. stratification, deformation) and igneous features (e.g. xenoliths, phenocrysts) close to this size may be either given undue importance or neglected. For example, the fine lamination in sample 19FR01 (Fig. 4H) was under-recognised by virtue of both the fragmentation of the sample and its covering by dust, requiring both CLUPI and HRC images to delineate the character and consistency of lamination. Contrastingly, the large olivine-rich xenolith in sample 09SV17 (Fig. 4B) was attributed over-importance by the 
geologists due to its accounting for a large portion of the core sample (particularly in the CLUPI image) which was not representative of the hand sample. Consequently, interpretations focussed on the mineralogy of this xenolith, and the porphyritic texture of the groundmass was ignored. In the third case, the large $(0.1-1 \mathrm{~cm})$ conglomeratic clasts in sample 11SP01 (Fig. 4E), were misidentified as alteration features due to the fact that they did not feature in the CLUPI image and were coarsely pixelated in the HRC image. The point of caution raised here is that neither CLUPI nor HRC is ideally adapted to imaging core characteristics in the sub-centimetre to centimetre range, and the identification of textural heterogeneity at these scales may be limited. Smaller and larger features and structures are more easily recognised, having constituent features that fall within the optimal resolution range of either CLUPI or PanCam at the contact operation working distance (see also Fig. 3). At this scale, determinations of granulometry are, in addition to being a function of resolution, also contrast-dependent: diverse mineralogies with distinct colour are easier to appraise in detail than homogeneous mineralogies in CLUPI and HRC images alone.

A second potential fabric misidentification involves the potential interpretation of damage resulting from the coring process as primary sedimentary features. Striations in sample 17NZ14 (Fig. 4C) were interpreted as primary bedding whereas, conversely, vesicles were thought to be mechanical erosion effects. Of note is that these features largely fall in the range of sub-centimetre to centimetre as discussed above. Striations are enhanced by the irregular drilling required to let the water flow along the core to evacuate fines, however, and would probably be less visible on ExoMars 2020 drill cores by virtue of its continuous drilling. Mineralogical data from Raman analyses can be used to revise interpretation, but this is nonetheless an important consideration since the imaging data have an inherent guiding value for geological interpretation. The fact that the numerous vesicles in sample 17NZ14 were not identified resulted in misinterpretation of its petrogenesis. The misidentification of conglomeratic clasts in sample 11SP01 (Fig. 4E) as alteration phenomena presents similar issues, however, atlases of texture at multiple scales (e.g. Schieber et al., 2007) may provide invaluable references for the interpretation of unusual or unexpected textures and microstructure in such cases.

\subsubsection{Analytical uncertainties}

Both of the above challenges imply that caution should be taken when determining lithology from core samples exhumed by ExoMars 2020, as neither analysed regions nor the core sample itself is necessarily representative of the bulk lithology. Although many units in Martian stratigraphy are relatively homogeneous on the metre-scale in mineralogical and sedimentological terms (McSween et al., 2009; Grotzinger et al., 2014; Quantin et al., 2016), this should not be assumed and small scale specificities have been key to palaeoenvironmental interpretation. In situ rover-based studies of Martian sediments in Gale Crater using ChemCam have identified, for example, centimetre-scale veins of light-toned, fracture-filling material determined to be the calcium sulphates gypsum and bassanite (Nachon et al., 2017). Rubin et al. (2017) also identified decimetre-scale cylindrical, pipe-like structures interpreted as fluidised sediment pipes in photographs taken by the Curiosity rover. Single analyses yielding either indicative or, conversely, unrepresentative data, for example the zircon identified using Raman spectroscopy in sample 19FR01, and suites of analyses 
failing to identify the characteristics of large portions of a sample, such as the dark laminations in the banded ferruginous chert 16GR01, introduce analytical uncertainties to lithological interpretations and therefore to estimations of the palaeoenvironment. For example, the calcium sulphate veins observed in Gale Crater, although volumetrically minor, have been critical in the proposition of post-depositional micro-environments (Kronyak et al., 2019) that may contribute to the alteration of biosignature preservation potential. Crosscorrelation of imaging and compositional data at both the outcrop and core scale is necessary and will rely on the multi-stage experimental cycle outlined in Figure 2 (outcrop study and core study) planned for the ExoMars rover mission (Vago et al., 2017). The PanCam wideangle cameras and CLUPI in the FOV1 operating mode will provide decisive complementary information at the surface. Further mitigation of residual issues in geochemical interpretation may be achieved through the combined approach of MicrOmega and RLS during the analytical stage, since MicrOmega will map the crushed grain surfaces and identify points of interest for further analysis by RLS (Bibring et al., 2017; Rull et al., 2017).

Among the most challenging mineralogies to identify, whether petrographically or spectroscopically, are clay minerals. This is evident from sample 17NZ05, in which the clay mineral matrix was not identified by any of the imagery and Raman spectroscopy conducted. Mineral phases immersed in clay mineral matrices tend to have their signals masked; indeed, even the identified minerals (leucite/labradorite and laboradorite/anorthoclase) exhibited either weak spectral signals or were identified only by certain unmasked peaks. This masking effect will be greater during the mission since the RLS instrument uses a $50 \mu \mathrm{m}$ laser and will likely incorporate multiple minerals into each analysis. At the outcrop scale and at the working distance from the mast-mounted instruments to the core sample, the Infrared Spectrometer for ExoMars (ISEM) instrument (Korablev et al., 2017) may be able to acquire compositional data relating to these phases both in the core and in the surrounding exposures. Axiomatic identifications of clay minerals will likely be achieved after MicrOmega analyses of crushed samples (Bibring et al., 2017). Furthermore, although a clay-rich core sample would lose its cohesion at the surface, its stratigraphy may nonetheless be inferred by Ma_MISS images within the borehole (De Sanctis et al., 2017) together with PanCam spectral images of the surrounding outcrops, before MicrOmega analyses.

The above considerations highlight that, since core samples may be neither wholly nor partly representative of their local and regional geological environments, the merging and integrative interpretation of data from all instruments of the ExoMars 2020 rover payload is necessary (Fig. 2). On Earth, the geological settings for ancient biosignatures are appraised on scales between regional and atomic (Wacey, 2009; Westall et al., 2015), and this can be achieved to some extent using the ExoMars 2020 instrumentation (Vago et al., 2017, 2018), which is split into panoramic, contact and analytical scales of observation. Within one stage of this protocol, for instance the contact analyses conducted herein, multiple complementary analyses of the same features or object may permit interpretation.

\subsubsection{Life detection}

A third category of challenges relates to biosignature detection. Macroscopic biosignatures, despite some controversies, are widely considered convincing evidence for life (Wacey, 2009), however, the range of biosignatures possible for Mars is expected to be 
subtler than that on Earth in terms of both morphology and geochemistry (Mustard et al., 2013; Westall et al., 2015; Hays et al., 2017). The challenge of life detection for ancient biosignatures on Earth (Westall et al., 2015; Hays et al., 2017), widely considered informative for the potential Martian biosphere, will be compounded on Mars by virtue of the reduced capabilities of rover instrumentation relative to Earth-based instrumentation. Microbial biosignatures were present in three of the samples studied: carbonaceous material in silicified volcaniclastic sediment 99SA13, biologically induced ferruginous precipitates in 16GR01, and organic-rich layers in 19FR01. In two cases (16GR01 and 19FR01), the presence of biology was correctly inferred, however, it was not explicitly identified, but rather implied from comparison to known rocks of similar origin with biological influences. Implication of biology in this manner could prove to be a significant drawback during the mission. Since even macroscopic traces of life are not wholly unambiguous, we suggest that some combination of optically identifiable biostructure and molecular characterisation is necessary to impute an unambiguous, in situ biosignature. We did not consider, however, in this blind test, the MicrOmega and MOMA data that will likely provide further important information on the composition of organic material in the samples. Were the crushed sample to contain bio-indicative molecules (e.g., Fig. 2, stage 2), closer analysis of the sample and locality in question would be necessitated. In the absence of either optically identifiable and/or molecular characterisation, the classification of putative biosignature or possible biosignature (sensu Buick, 1990) should be used. Such a potential biosignature might be appraised by Mars sample return.

\subsection{Complementarity of the ExoMars 2020 instrumentation}

Our blind test demonstrated that rover imaging instrumentation alone can, in many instances, make a reasonably comprehensive geological analysis of samples of limited size, such as the ExoMars 2020 core samples $(3 \times 1 \mathrm{~cm})$. Accurate identifications were made for four of the tested samples, and many informative characteristics noted for two others. Two samples (17NZ14 and 11SP01) were inaccurately identified, although, even in these cases, informative observations were made. In all cases, confidence in interpretation was significantly increased when mineralogical data (Raman spectra) were added. Mineralogical data can confirm interpretations based on optical imagery and, where petrographic mineral identification is ambiguous, add key information that allows the lithology to be characterised. The combination of HRC and CLUPI core images identified material properties (e.g., friability, hardness, cohesion) that inform lithological interpretation even in the absence of mineralogical data. In most cases, the combination of optical and mineralogical data led to an accurate estimation of the identity of the sample. Instrumental collaboration is therefore of fundamental and decisive importance in the success of the ExoMars 2020 mission (Figs. 2-3), since the mission is constrained by the small sample sizes inherent to its innovative approach. The interrelationship of rover instrumentation, and the possibility to ascertain consistency between observations during the panoramic, contact and analytical stages, will provide guidance and validation for all ExoMars 2020 findings (Vago et al., 2017). Such progressive learning has been key to the interpretation of samples taking into account the strengths and shortcomings of previous rover instrumentation suites (Mangold et al., 2017). In the case of ExoMars 2020 rover operations, petrographic studies using PanCam, HRC, ISEM, and 
CLUPI images in their panoramic and contact operating modes should guide the selection of samples for analytical study. Any indication of deleterious secondary alteration, for instance veining, fracturing, oxidation or other chemical weathering (even if misidentified as in the case of 11SP01), might constitute a case against further assessment of a potentially biosignature-bearing sample. Contextualising information at each sampling site by the ExoMars 2020 panoramic operations should be the primary safeguard against incorrect interpretation.

\subsection{Strategies for geological appraisal of core samples}

Our protocol involved a checklist of lithological characteristics (Tables 2-3), and largely achieved its goals by guiding the questioned geologists in their answers, however, a reluctance to offer additional comments on the samples in an open space on the response form meant that many sample-specific characteristics were ignored, for example grain morphologies indicating potential transport agents, and sedimentary observations beyond stratification. Since it is known that grain morphology can be an indicator of transportation mode (Williams et al., 2013; Kapui et al., 2018), and since this can be determined in mediumcoarse sandstones at CLUPI resolutions (Fig. 3), such sedimentary characters may assist palaeoenvironmental reconstructions. Sedimentology has proven invaluable in the reconstruction of other Martian localities, most notably the varied deposits of Gale Crater (Grotzinger et al., 2014; Schieber et al., 2017; Banham et al., 2018) and across Meridiani Planum (Squyres et al., 2005; Grotzinger et al., 2005; McLennan et al., 2005). Although methods of calculating the probability of biosignature detection and biogenicity on Mars have been extensively proposed (Westall et al., 2015; Vago et al., 2017; Neveu et al., 2018), these methods usually do not explicitly characterise habitability in its geological context. Westall et al. (2015) considered the processes from origination to transportation in trajectories of biosignature evolution, a key parameter of which is the geological setting of the primary biosignature, defined by sedimentological and igneous characteristics that, during the ExoMars 2020 mission, will be evaluated. Vago et al. (2017) 'scored' aqueous and hydrothermal habitats within their biosignature calculator, the evidence for which will come from individual morphological and geochemical datapoints. The framework for biosignature plausibility, detection and proof must evidently encompass geospheric and potential biospheric notions, an approach considered in terms of the co-evolution of Martian habitats and biotopes by Westall et al. (2015) and Cabrol (2018).

In this blind test, surface geological context, i.e., whether the core sample is representative of the outcrop sample, was not considered. We therefore focussed on the critical importance of steps 3-5 in the ExoMars 2020-specific workflow shown in Figure 2. This represents a worst-case scenario for sample identification since, although this challenging situation is entirely possible on Mars, it is more likely that at least some informative guidance will be provided by surface lithology and characteristics of the landscape.

Our protocol explicitly requested that no speculative interpretation be made in the absence of data. Mineralogical data were generally able to overturn incorrect interpretations made based on imaging data alone, however, the combination of HRC and CLUPI core imaging, together with outcrop data gathered in the initial stages of mission experimental 
cycles, would likely place firm constraints on local geological conditions and suggest the likelihood of habitability. The progressive arrival of data from the panoramic, contact and analytical stages of ExoMars 2020 rover operations has many advantages. As shown in this blind test, increasing the amount of data available to scientists generally resulted in continually more accurate interpretations. From a geological standpoint, the progressive arrival of data closely aligns the process of interpretation with the traditional 'terrestrial' approach. Panoramic observations are approximately equivalent to the planning and preliminary stages of fieldwork in a new location. Contact operations reflect the main stage of fieldwork: outcrop examination and sampling. The analytical stage of operations is analogous to laboratory work when the field campaign is complete. We consider that the progressive arrival of data, although potentially demanding repeated reinterpretation, should not be considered as a negative aspect of the protocol, but rather an opportunity for the construction of hypotheses and their evaluation and modification in light of increasingly specific datasets.

\section{6) Conclusions}

In this contribution, we have sought to demonstrate and evaluate the challenges that will be faced in the interpretation of the $3 \times 1 \mathrm{~cm}$ core samples exhumed by the ExoMars 2020 rover. Cores should be imaged by the PanCam HRC and CLUPI during the brief period before their ingestion and crushing within the body of the rover. Geological appraisals of these cores will provide fine-scale context information for potential organic signatures detected within. Core samples may not be representative of surface lithology and, in this worst-case scenario, accurate geological descriptions of core samples will be required to provide fine-scale characterisation for the petrological context of putative biosignatures. Our findings and recommendations are as follows:

- Detailed, accurate petrology - and by extension a potential estimate of habitability - is often possible using CLUPI and HRC images alone. Since different resolutions and scales of observation are encountered in HRC and CLUPI images, there are often opportunities to rectify misinterpretations based on scale-representativeness.

- Compositional data from the analytical laboratory instruments alone are not sufficient to characterise the petrogenesis and petrology of a rock; we therefore recommend that imaging cores using both HRC and CLUPI should form an integral part of the contact operations of the ExoMars rover and form a framework for the interpretation of analytical data. Furthermore, our results confirm the necessity of multi-scalar, multi-instrument analyses during the rover mission and the opportunities for progressive learning that they permit.

- It is more challenging to determine petrological character from a core sample than from a hand sample or from direct outcrop data. Core sample interpretation has a number of shortcomings, which vary between rock types. Sample heterogeneity, distinguishing subcentimetric to centimetric structures, and the unambiguous identification of biosignatures are amongst the challenges most often faced due to the limited information available in 
high-resolution images. Shape-relevant challenges to sample interpretation should be taken into account when conducting image analysis.

- Mineralogical (compositional) data improved confidence in, and accuracy of, petrogenetic interpretations, and a synergistic approach advocating the collaboration of the panoramic, contact and analytical onboard instrumentation will be essential to achieve the scientific goals of the mission. 